\title{
Tangence
}

\section{Réécriture filmique et discours sur l'immigration}

\section{Le gone du Chaâba d'Azouz Begag et de Christophe Ruggia}

\section{A Film Rewriting and Discourse on Immigration}

\section{Le gone du Chaâba by Azouz Begag and Christophe Ruggia}

\section{Alexie Tcheuyap et Étienne-Marie Lassi}

Numéro 75, été 2004

Les formes transculturelles du roman francophone

URI : https://id.erudit.org/iderudit/010783ar

DOI : https://doi.org/10.7202/010783ar

Aller au sommaire du numéro

Éditeur(s)

Tangence

ISSN

1189-4563 (imprimé)

1710-0305 (numérique)

Découvrir la revue

Citer cet article

Tcheuyap, A. \& Lassi, É.-M. (2004). Réécriture filmique et discours sur l'immigration : Le gone du Chaâba d'Azouz Begag et de Christophe Ruggia.

Tangence, (75), 41-62. https://doi.org/10.7202/010783ar
Résumé de l'article

Le gone du Chaâba d'Azouz Begag illustre la difficile cohabitation entre les immigrants maghrébins en France et leur communauté d'accueil. Onze ans après sa publication, Christophe Ruggia réalise une version filmique du roman, déplaçant ainsi du livre à l'écran la problématique de l'immigration. Cet article, qui s'intéresse aux modalités esthétiques et discursives de cette réécriture, identifie et analyse quelques mutations textuelles significatives afin de déterminer quels suppléments de sens différentiels elles apportent au texte tuteur dont l'altération semble inévitable dès que se met en marche le procès de la reprise. Ces diverses transformations, opérées souvent sous le mode de l'hypertrophie, permettent de reformuler le discours romanesque sur l'identité et les trajectoires sociales des populations immigrées. 


\title{
Réécriture filmique et discours sur l'immigration. Le gone du Chaâba d'Azouz Begag et de Christophe Ruggia
}

\author{
Alexie Tcheuyap et Étienne-Marie Lassi, \\ Université de Calgary
}

Le gone du Chaâba d'Azouz Begag illustre la difficile cohabitation entre les immigrants maghrébins en France et leur communauté d'accueil. Onze ans après sa publication, Christophe Ruggia réalise une version filmique du roman, déplaçant ainsi du livre à l'écran la problématique de l'immigration. Cet article, qui s'intéresse aux modalités esthétiques et discursives de cette réécriture, identifie et analyse quelques mutations textuelles significatives afin de déterminer quels suppléments de sens différentiels elles apportent au texte tuteur dont l'altération semble inévitable dès que se met en marche le procès de la reprise. Ces diverses transformations, opérées souvent sous le mode de l'hypertrophie, permettent de reformuler le discours romanesque sur l'identité et les trajectoires sociales des populations immigrées.

La «littérature de l'immigration» se définit surtout par un contexte socioculturel de production situé en terre étrangère. Il s'agit en réalité d'une littérature "apatride» en ce sens que sa nonintégration dans la littérature nationale de la «terre d'accueil» est à l'image du type d'exclusion que connaissent les acteurs à l'œuvre dans ces productions. Ce "malaise» dans la définition est encore visible dans la terminologie généralement employée: littérature émergente, écriture décentrée, littérature «ethnique» ou minoritaire. Ce lexique implique une problématique spécifique que soustend la mise en contact de plusieurs cultures. L'immigrant ${ }^{1}$ arrive,

1. Nous aimerions noter ici que, dans cette étude, le terme immigrant désigne d'abord et avant tout une personne d'origine étrangère. Certes, aussi bien 
pétri d'un ensemble de valeurs, d'une vision du monde et d'un habitus différents de ceux de la communauté d'accueil. Comme le relève Lévi-Strauss ${ }^{2}$, bien que les cultures se fécondent et s'enrichissent par leurs différences, le propre de chacune d'elles est, tout en s'ouvrant aux autres, de résister à leurs influences pour se préserver de l'uniformisation. On en déduit assez aisément la position de l'immigrant, dont L'aventure ambiguë de Cheikh Hamidou Kane ${ }^{3}$ avait déjà illustré une dimension. Pris entre l'angoisse de la séparation et l'espoir de l'arrivée, la peur de l'exclusion et l'appréhension de l'intégration, la volonté de rester soi et la fascination de l'Autre, l'immigrant doit de surcroît faire face au regard du groupe d'accueil qui, parfois, le rejette. La littérature de l'immigration se caractérise donc essentiellement par la prise en charge de ces contraintes socioculturelles et on comprend que les questions d'identité et de représentation apparaissent comme sa principale préoccupation.

Le premier roman d'Azouz Begag, Le gone du Chaâba ${ }^{4}$, appartient à cette catégorie d'œuvres désignées en France par «littérature beur», formule qui désigne les œuvres produites par les descendants de l'immigration maghrébine en France. Selon Martine Delvaux, ces romans, et on le verra avec celui de Begag, se définissent par «un double mouvement d'éloignement et de rapprochement, d'appartenance et de désappartenance ${ }^{5} »$. Le gone $d u$ Chaâba raconte, sur un mode autobiographique, l'itinéraire d'un "gone», c'est-à-dire d'un gamin, fils d'immigrant algérien dans une banlieue de Lyon. Le roman illustre les difficultés de la cohabitation entre cette culture arabe maghrébine minoritaire et la culture française dominante, en même temps que les stratégies développées de part et d'autre pour les surmonter. Il s'agit donc,

dans le roman que dans le film, il est clairement indiqué que le petit Azouz (Begag) est «français ». Mais il est évident, au vu de l'espace dans lequel il vit avec les autres acteurs et du traitement particulier dont ils bénéficient de la part de l'administration, que la citoyenneté française est une simple formalité administrative qui confère plus de devoirs et génère plus de frustrations que de droits. Et on peut constater facilement que la «francité» des populations du Chaâba est toute singulière. Il est aussi important de rappeler que, tant dans le roman que dans le film, les personnages sont tous algériens, arabes et musulmans. Il n'y a même pas de Berbères, ni de ressortissants d'un autre pays du Maghreb.

2. Voir, à ce propos, Claude Lévi-Strauss, De près et de loin, Paris, Seuil, 1990.

3. Cheikh Hamidou Kane, L'aventure ambiguë, Paris, Julliard, 1961.

4. Azouz Begag, Le gone du Chaâba, Paris, Seuil, 1986.

5. Martine Delvaux, "L'ironie du sort. Le tiers-espace dans la littérature beur", The French Review, vol. 68, 1995, p. 683. 
dans ce roman, du discours d'un immigrant sur l'immigration. Mais que devient ce discours à l'écran? Voilà une question qui mérite certainement que l'on s'y intéresse.

De fait, onze ans après la publication du roman, Christophe Ruggia réalise une version filmique du Gone du Chaâba, déplaçant ainsi du livre à l'écran la problématique de l'immigration. Quelles sont les modalités de cette adaptation filmique? La tâche de Ruggia s'est-elle limitée à transposer un contenu ou un message d'un code à l'autre? Auquel cas il souscrirait à cette tradition théorique qui veut que toute adaptation filmique soit une "fidèle» retranscription du texte littéraire, une migration du discours littéraire vers l'écran puisque, selon André Bazin, «la bonne adaptation doit parvenir à restituer l'essentiel de la lettre et de l'esprit ${ }^{6} »$. C'est là, du moins, ce que donne à penser l'analyse qu'a faite de ce film Alec G. Hargreaves, qui relevait déjà que, «bien qu'il y ait quelques changements dans le film, quand on le compare avec le roman, il est largement fidèle au texte de Begag, par-dessus tout à la primauté qu'il accorde aux expériences et au point de vue du jeune Azouz, appelé Omar dans le récit filmique ${ }^{7}$ ».

Nous nous proposons de déplacer la réflexion pour la situer essentiellement dans une perspective herméneutique. Ce n'est plus la question de la "fidélité», concept improbable et fort incertain, que nous entendons interroger, car nous estimons que toute réécriture, qu'elle ait lieu dans le même média-genre ou d'un médiagenre à un autre, est toujours l'occasion de métamorphoses multiples, ainsi que l'ont indiqué les travaux déjà anciens de Gilles Deleuze $^{8}$ ou des travaux aussi récents que ceux de Kamilla Elliot ${ }^{9}$, James Naremore ${ }^{10}$ ou Alexie Tcheuyap ${ }^{11}$. Nous considérons donc

6. André Bazin, Qu'est-ce que le cinéma? tome 2: Le cinéma et les autres arts, Paris, Le Cerf, 1959, p. 21.

7. Alec G. Hargreaves, «Resuscitating the Father: New Cinematic Representations of the Maghrebi Minority in France», Sites. Journal of the Twentieth Century Contemporary French Studies, Oxford, vol. 4, n ${ }^{\circ}$ 2, 2000, p. 345: "although there are some changes in the film, compared with the original narrative, it is broadly faithful to Begag's text, above all in the primacy which it accords to the experiences and viewpoint of the young Azouz, renamed Omar in the movie».

8. Gilles Deleuze, Différence et répétition, Paris, Presses universitaires de France, 1968.

9. Kamilla Elliot, Rethinking the Novel/Film Debate, Cambridge University Press, 2003.

10. James Naremore (sous la dir. de), Film Adaptation, New Brunswick, Rutgers University Press, 2000.

11. Alexie Tcheuyap, "Le Texte littéraire à l'écran. Approches et limites théoriques ", Protée, Chicoutimi, vol. 29, n 3, hiver 2001-2002, p. 87-96. 
ces métamorphoses inévitables, mais, surtout, nous choisirons celles qui nous paraissent significatives et nous en analyserons les enjeux. Autrement dit, nous identifierons des mutations textuelles et verrons quelle en est la portée, quels suppléments de sens différentiels elles apportent au texte tuteur dont l'altération semble inévitable dès que se met en marche le procès de la reprise. En outre, on verra que les diverses transformations, opérées souvent sur le mode de l'hypertrophie, permettent de reformuler le discours romanesque sur l'identité et les trajectoires sociales des populations immigrées, comme le montrent les deux versions du Gone du Chaâba qui, du roman au film, témoignent de transformations notables sur le plan de la narration.

\section{Les métamorphoses de la narration}

Les deux versions du Gone du Chaâba ont bel et bien des aspects en commun. Ce qui frappe d'emblée, c'est le mode d'énonciation autobiographique, le roman et le film se présentant comme le récit de la vie d'un enfant, immigré algérien de la deuxième génération qui cherche à se frayer un chemin dans la société française des années 1960. Le romancier le nomme Azouz et lui confie la narration à la première personne. Le réalisateur l'appelle Omar, le montre au début du film, un livre ouvert à la main et, à la fin, écrivant péniblement dans un cahier d'écolier «les mémoires du Chaâba». La voix over, celle d'un enfant, se veut la lecture, par Omar lui-même, du texte qu'il a écrit. De cette manière, Ruggia, comme Begag dans le roman, laisse le jeune protagoniste assumer la narration. Cette voix du fils, ainsi que l'indique Hargreaves, contrôle l'espace narratif et discursif, ce qui concourt à presque effacer la présence de son père. Mais davantage dans le film que dans le roman, l'autorité narrative revient à l'enfant. La naïveté, somme toute compréhensible, du regard, des gestes et des commentaires du petit narrateur, conserve au film l'humour déjà perceptible dans le roman, sans masquer pour autant la gravité des situations rapportées.

En effet, Le gone du Chaâba, roman et film, est une chronique relatant les événements au fur et à mesure qu'ils surviennent. On a ainsi droit à une intrigue linéaire qui mène les protagonistes d'un espace périphérique à l'autre, plus près du centre de la ville, d'une condition d'insalubrité et de misère abjectes à des conditions d'existence certes imparfaites mais améliorées, d'une situation 
d'ignorance totale à une situation de relatif savoir. L'axe temporel du récit ne souffre d'aucune digression, à l'exception de la séquence de la circoncision, analeptique dans le roman, mais replacée dans l'ordre chronologique normal dans le film. Il s'agit, dans un cas comme dans l'autre, d'un récit de formation où les épreuves rencontrées sont autant d'occasions d'apprentissage et l'équation de la fin du film entre Azouz Begag et Omar viserait à soutenir cette impression, pour ainsi dire, de Bildungsfilm.

La voix narrative et la construction de l'intrigue, on le voit, établissent un parfait parallèle entre le film et le roman. On a l'impression que le réalisateur déploie un effort réel pour maintenir dans son film les mêmes événements, avec parfois le même ordonnancement, la même thématique et la même atmosphère que dans le roman, et conserver par conséquent au cinéma tout ce qui caractérise, d'après Régine Robin du moins, les littératures émergentes ou minoritaires ${ }^{12}$. De là, on pourrait croire que les modifications subies par le texte lors du passage du littéraire au filmique tiennent de la différence entre les deux médias, l'outil cinématographique pouvant en même temps accueillir et faire écran à la fabula littéraire.

En effet, le roman n'est pas soumis aux mêmes contraintes de temps que le film et peut se répandre en commentaires et en descriptions. Au cinéma, en revanche, le temps est un paramètre incontournable. La durée habituelle d'un long métrage comme Le gone du Chaâba est de 90 minutes: du roman au film, l'histoire s'allège donc considérablement, le réalisateur l'ayant élaguée de nombreux éléments. Comme la compression temporelle est importante, le réalisateur doit alors reconstituer les protocoles narratifs pour assurer cette fonction du récit, fondamentale selon Christian Metz et qui permet «de monnayer un temps dans un autre temps ${ }^{13} »$. Cette compression résulte non seulement de contraintes propres au média filmique, qui doit limiter le récit à un nombre précis de minutes, mais aussi de choix délibérés qu'opère le réalisateur, qui abrège ou amplifie certains passages du texte.

12. Régine Robin («Présentation», Études littéraires, Québec, vol. 29, n ${ }^{\text {os }} 3-4$ (L'ethnicité fictive: judéité et littérature), hiver 1997, p. 7) cite, entre autres critères pour caractériser les littératures émergentes, l'écriture autofictionnelle, l'implication communautaire et la problématisation de l'identité.

13. Christian Metz, Essais sur la signification au cinéma, Paris, Klincksieck, 1968, p. 28. 
En effet, le temps de l'histoire se trouve réduit de plus de la moitié. Ruggia situe l'action de son film entre 1965, année qui marque le troisième anniversaire de l'indépendance de l'Algérie, et le 5 août 1966, date de l'emménagement à Lyon de la famille Bouzid, qui quitte alors le Chaâba, c'est-à-dire le «ravin» ou, pour mieux dire, le "dépotoir où l'on déverse les poubelles", suivant le sens de ce mot arabe. Le roman ne précise pas le début exact, mais s'étend jusqu'en 1968. À l'évidence, certains moments décisifs de la vie du narrateur sont passés sous silence dans le film: en l'occurrence, son séjour dans le nouveau quartier, à l'école Sergent Blandau et au lycée Saint-Exupéry. Ce faisant, certaines figures ayant profondément marqué la vie du jeune héros sont effacées: Madame Valard, sa maîtresse de CM2, Monsieur Émile Loubon, son professeur de français en classe de sixième, les frères Taboul d'origine juive, Alain, «l'immigré de la tour Eiffel», ses camarades de classe, ne font pas partie, en effet, de l'univers du film. Louise, la seule Française du Chaâba, initiatrice de la guerre contre les prostituées, disparaît elle aussi.

Mais le réalisateur ne se limite pas à la classique économie narrative caractérisant tout passage à l'écran. En plus de la suppression, Ruggia procède aussi à une compression des séquences et des personnages. Des scènes éparses dans le roman sont rassemblées dans le film en une seule séquence et, de celles longuement développées à l'écrit, le réalisateur ne retient quelquefois qu'un aspect, le début ou l'aboutissement. Par exemple, économie est faite de toutes les maladresses linguistiques et comportementales du narrateur dans la classe de monsieur Grand. On le présente comme un élève déjà adapté et brillant, et si chacun de ses succès est célébré en son temps dans le roman, le film les résume en une seule séquence, celle où Bouzid lui fait des confidences sur la providence et manifeste son désir d'inviter le professeur à dîner. De même, les préparatifs de la guerre contre les prostituées sous la houlette de Louise ne figurent pas dans le film, et seules sont exposées les attaques des gones et l' «armistice» conclu par Rabah.

Quant aux personnages, quelques-uns cumulent des rôles autrement distribués dans le roman. C'est le cas de Bouchaoui, à qui l'on attribue les actions de différents protagonistes dans le roman: celles d'Ali, l'oncle d'Azouz/Omar qui lui vide sur la tête le contenu de son pot de chambre, et celles de Berthier, collègue français de Bouzid et ancien propriétaire du Chaâba, dont la visite tardive perturbe le sommeil de la famille. Massaoui, camarade de classe d'Azouz, rebelle et nul, est supprimé et son rôle se trouve 
redistribué entre Hacène et Sélim Bouchaoui. Ceux-ci, du coup, se trouvent "surfonctionnalisés» du fait du passage à l'écran qui réduit, en raison de contraintes financières ou esthétiques, le personnel du récit. La surcharge actantielle donne aux personnages maintenus, lesquels ne sont toutefois pas les plus importants du texte romanesque, une signification autrement plus importante dans la marche narrative. Toutes ces opérations de suppression et de compression, si elles raccourcissent le récit, lui donnent surtout une nouvelle configuration, de même qu'elles confèrent aux personnages une nouvelle consistance, créant du coup un décalage fort significatif entre les deux textes.

En effet, du roman à l'écran, ce sont surtout des personnages français qui ont disparu, le film ne gardant que quelques figures françaises: M. Grand, le maître, Jean-Marc Laville, une prostituée et les huit policiers, ce qui est curieux pour un film dont l'action se situe en France. Comme par miracle, le pays hôte, lieu de toutes les contradictions, de tous les conflits, mais aussi de tous les espoirs pour les immigrés, tend à s'effacer dans le récit filmique. Au reste, la plupart des personnages supprimés ont en commun leur statut de marginal: Berthier est maçon, comme Bouzid, et ancien propriétaire du Chaâba; Louise habite le Chaâba et, bien qu'elle y passe pour une représentante de l'élite, manifeste des signes de retard mental et ne se prive pas des trésors de la poubelle; Loubon est né en Algérie, y a séjourné pendant longtemps et a une parfaite maîtrise de la langue et de la culture arabes. Malgré leur appartenance à la culture française dominante, ces personnages ne peuvent pas se prévaloir de la même vision du monde que leurs compatriotes. Ayant fait l'expérience de l'émigration, soit à l'occasion d'un voyage, soit en raison de leur déclassement social, ils jouissent d'un horizon plus large et donc de la capacité «de relativiser et de se relativiser là où les autres sont en proie aux ornières de la monovalence ${ }^{14}{ }^{\prime}$. On a l'impression, en somme, que le réalisateur du film a voulu donner de la France une représentation monolithique, débarrassée de toute idiosyncrasie. L'effacement des Juifs, de madame Valard et du Parisien serait justifié par la même préoccupation. En ce sens, M. Grand, sa classe et les policiers sont la représentation métonymique de l'Hexagone et de ses institutions: leurs rapports avec la communauté immigrée, même si elle est «française», comme le clame par moment avec fierté le jeune

14. Julia Kristeva, Étrangers à nous-mêmes, Paris, Fayard, 1988, p. 16. 
narrateur, se réduisent à la répression et à l'assimilation la plus brutale. Cette représentation filmique, qui nuance le mythe du paradis hexagonal, contribue à apporter au film, comme à toute écriture beur, "un angle de vue décentré par rapport à tout ce qui se dit sur les immigrés et la société française ${ }^{15}$ ».

Les personnages maghrébins sont, en revanche, traités autrement, mais on aboutit au même résultat. Par une caractérisation à vocation métonymique, le film présente trois familles algériennes à la faveur d'une subtile mise en parallèle de trois enfants et de leurs pères. Omar, Hacène et Bouchaoui, dont les attitudes différentes sont attribuables à la nature de l'éducation reçue, parviennent à propulser leurs familles au devant de la scène, reléguant dans un rôle de figurant les autres dont ils sont quand même une émanation. Bref, les compressions et les suppressions des séquences et des personnages n'ont eu d'effet, on le constate, que sur la durée de l'histoire. Elles affinent les antagonismes socioculturels en définissant plus clairement et plus distinctement que dans le roman le statut de la France, qui incarne une culture dominante, et celui des Maghrébins, enfants d'une culture dominée. En mettant l'accent sur l'institution scolaire, lieu d'apprentissage de la culture, et sur l'«institution" policière, instance de la censure des écarts, Ruggia situe la France comme groupe de référence, selon la terminologie de Landowski ${ }^{16}$, qui seul édicte les critères d'admission ou d'exclusion, afin de sauvegarder son identité face à une culture étrangère dont les représentants, bien qu'aussi jaloux de la leur, recherchent malgré tout une certaine reconnaissance. Le réalisateur met ainsi en évidence l'un des problèmes les plus importants de l'immigration: la marginalisation de l'immigrant, perçu comme autre et différent. On comprend alors pourquoi l'altérité est si difficile à assumer.

\section{Altérité et topographie de l'immigration}

Le cinéma a cette possibilité de montrer ce que même le roman n'évoque pas toujours. C'est le cas pour ce qu'on peut considérer comme les marqueurs de l'altérité, lesquels permettent d'identifier ce qu'on appelle parfois par euphémisme des "minorités visibles»,

15. Azouz Begag et Abdelatif Cahaouite, Écarts d'identité, Paris, Seuil, 1990, p. 100.

16. Éric Landowski, Présences de l'autre, Paris, Presses universitaires de France, 1997, p. 45-86. 
qui sont en général aussi des "minorités audibles». La communauté maghrébine dans le film de Ruggia se distingue par les traits physiques de ses membres, leur religion, leurs habitudes vestimentaires, culturelles et linguistiques - et surtout leur statut social. Les Maghrébins se démarquent des Français par les traits de leur visage, leurs cheveux lisses et noirs, les tatouages pour les hommes et le maquillage pour les femmes. La distinction physique est très importante dans le film, l'expression "tête d'Arabe», qui fait écho à celle de "tête de Turc», étant souvent utilisée par les jeunes Arabes eux-mêmes comme signe d'appartenance et, par les autres, comme critère d'exclusion et de dérision. Le réalisateur en rend compte par la position rapprochée de la caméra. On obtient ainsi des gros plans sur ces marques de l'arabité, comme le tatouage sur la joue droite d'Omar ( $32^{\mathrm{e}}$ et $59^{\mathrm{e}}$ minutes) ou le point noir sur le front de madame Bouafia ( $34^{\mathrm{e}}$ minute). Ces signes peuvent paraître bien dérisoires, mais ce sont en général ceux-ci qui constituent les éléments d'identité et d'identification, lesquels génèrent souvent les différentes scènes de discrimination que l'on retrouve dans le roman et le film.

Les habits sont un autre signe extérieur de "l'étrangeté» des personnages d'origine maghrébine. À l'occasion de la fête de la circoncision, rituel religieux musulman, la caméra focalise sur les gandouras et les robes richement brodées, comme pour bien signifier que ces vêtements de circonstance sont le signe apparent d'une particularité plus profonde. On a l'impression qu'il s'agit de montrer que l'écart sur le plan vestimentaire n'est rien d'autre que le signifiant d'un écart culturel. De fait, par les gros plans sur les signes distinctifs extérieurs et certaines pratiques culturelles, le réalisateur en arrive à construire une image de la communauté maghrébine comme groupe homogène jouissant d'une identité propre. Dès lors, les structures du récit s'organisent de façon binaire avec, d'un côté, les Arabes, et, de l'autre, les Français.

C'est précisément ce qu'illustre la construction de l'espace. Deux micro-espaces structurent le film et le roman: le Chaâba et l'école. Nous découvrons le Chaâba à travers les panoramiques qu'en donne le réalisateur en accompagnant Omar et les gones dans leurs promenades et leurs jeux. C'est un tableau dégoûtant qui se déploie à notre vue: des huttes faites de matériaux de fortune et construites sans aucun souci esthétique, des allées gluantes de saleté, des flaques d'eau, une bande d'enfants abandonnés et courant dans tous les sens, voilà l'essentiel du décor. C'est une enceinte close, un véritable îlot d'insalubrité séparé de la 
ville par deux frontières naturelles, la forêt et le Rhône, et deux frontières artificielles, la grille métallique et le boulevard. Cet univers a ses hommes et ses pratiques. Quoique situé en France, le Chaâba, aussi bien dans le roman que dans le film, et peut-être plus dans ce dernier qui montre ce que le verbe ne décrit pas toujours, correspond exactement à ce que Frantz Fanon relevait de l'espace occupé par «l'indigène» dans la ville coloniale:

La ville du colonisé, ou du moins la ville indigène, le village nègre, la médina, la réserve est un lieu malfamé, peuplé d'hommes malfamés. On y meurt n'importe où, n'importe comment. On y meurt n'importe où, de n'importe quoi. C'est un monde sans intervalles, les hommes y sont les uns sur les autres, les cases les unes sur les autres. La ville du colonisé est une ville affamée, affamée de pain, de viande, de chaussures, de charbon, de lumière. La ville du colonisé est une ville à genoux, accroupie, une ville vautrée ${ }^{17}$.

Les habitants du Chaâba sont certes des "Français », mais surtout des immigrés dont la condition n'est pas des plus enviables dans la communauté d'accueil. Leur niveau de vie, de même que leur origine, leur imposent une certaine attitude. Tous sont des travailleurs manuels survivant à peine et aucun ne peut s'offrir le luxe d'une maison bien compartimentée. Eau courante, salle de bain ou électricité, tout y manque: salle de séjour, chambre à coucher et cuisine y sont confondues. Sorte de bantoustan hexagonal, le Chaâba est un lieu de promiscuité absolue, où l'on fait la queue pour utiliser l'unique latrine à tour de rôle, où les enfants s'épient et épient leurs parents, faisant de l'intimité conjugale une utopie. La caméra effectue des plans longs sur les gestes quotidiens tels que le bain matinal de Bouzid, la lessive tumultueuse à la pompe, le repas familial, l'abattage des moutons, etc., comme pour mettre au jour les activités qui singularisent les habitants du bidonville.

L'autre espace est l'école, plus particulièrement la classe de M. Grand. Elle apparaît comme le contrepoint du Chaâba. Les mouvements de la caméra qui soulignent à grands traits l'insalubrité et l'immoralité là-bas focalisent ici sur les coins d'un tableau où sont inscrites des règles d'hygiène et de morale. Les leçons de M. Grand insistent de manière obsessionnelle sur la morale et la propreté, relevant le divorce radical entre ses enseignements et les

17. Frantz Fanon, Les damnés de la terre, Paris, Maspéro, 1961, p. 8. 
pratiques du bidonville. Bouchaoui exprime bien cette différence lorsqu'il déclare, réagissant à la réponse d'un élève qui dit embrasser son père chaque soir avant d'aller au lit: «Si je fais ça, mon père appelle la police».

Les prises de vue montrant M. Grand (son nom est très significatif à cet égard) le placent toujours dans une position panoptique permettant de visualiser des scènes du roman. Le plus souvent filmé en contre-plongée ou en plan rapproché, il domine la scène, surveille et rappelle à l'ordre. Mis en valeur par la caméra, son discours s'en trouve valorisé d'autant et ses leçons de morale et d'hygiène deviennent «l'inventaire des traits différentiels qui serviront à construire "les figures de l'autre"; construction qui produit souvent des systèmes de ségrégation ${ }^{18} »$. Dès lors, les traits identitaires relevés plus haut cessent d'être de simples signes dénotant la différence et se voient affectés de significations nouvelles en devenant autant de marques de normalité ou d'anormalité.

La présentation que donne Ruggia de l'école de M. Grand fait glisser corrélativement les termes Arabe et Chaâba du domaine du vocabulaire objectif à celui du vocabulaire subjectif, pour reprendre la terminologie de Kerbrat-Orecchioni ${ }^{19}$. Ils ne se contentent plus de désigner un peuple ou un lieu, ils se chargent aussi d'exprimer les sentiments de celui qui les profère. Bâti sur un remblai qui sert de décharge publique, le Chaâba symbolise la saleté. C'est le lieu où l'on se débarrasse des rebuts et les Maghrébins qui y trouvent leur subsistance deviennent, par ricochet, des rebuts de la société. Ils sont tenus à l'écart de celle-ci, tels des parias, et il est intéressant de remarquer que, dans le film, le flux des personnes se fait en sens unique, du Chaâba vers l'école et vers la ville. Une fois la barrière du bidonville franchie, les gones se baissent tous et nettoient leurs chaussures maculées de boue, comme pour se purifier des souillures de leur origine avant de continuer leur marche. Au-delà de la nature coloniale de cette topographie, ce qu'il importe de relever concerne aussi le rapport d'exclusion qui structure l'espace: d'une part, le Chaâba et, de l'autre, l'ailleurs que représente cette France dont la présence reste fantomatique dans le film. Tout se passe comme si ce lieu habité

18. Janet M. Paterson, "Pour une poétique du personnage de l'autre», Texte, Toronto, nº 23-24, 1998, p. 106.

19. Catherine Kerbrat-Orecchioni, L'énonciation de la subjectivité dans le langage, Paris, Armand Colin, 1980. 
par les immigrés était un lieu maudit, une sorte de prison d'où ils ne pourraient partir. Le pays d'accueil devient du coup «[e]space découpé, immobile. Chacun est arrimé à sa place. Et s'il bouge, il y va de sa vie, contagion ou punition ${ }^{20} »$.

Par ailleurs, on a le sentiment que l'impureté associée au bidonville se manifeste sur le plan linguistique par la connaissance approximative de la langue française. Les Maghrébins parlent un français «corrompu» par la phonologie arabe et par une mauvaise connaissance de la syntaxe et du vocabulaire. Bien que le film n'y insiste pas autant que le roman, il montre quand même l'acharnement de $\mathrm{M}$. Grand à imposer à ses élèves une bonne maîtrise du français: de fait, en plus de l'hygiène et de la morale, ses leçons portent uniquement sur la langue.

On pourrait relever enfin l'impureté morale. Le Chaâba, dans le roman comme dans le film, est associé à l'idée de malhonnêteté et d'illégalité. Les ordures, déversées et acclamées comme une fête dans le bidonville, sont surmontées d'une plaque interdisant tout dépôt de saleté. De même, c'est aux encablures du Chaâba que les péripatéticiennes viennent exercer leur commerce illicite. Les restes du centre de la cité sont déversés à la périphérie. Les déchets ménagers rejoignent les déchets humains, toujours en proie par ailleurs au brigandage, à l'immoralité et à la criminalité. Autrement dit, la construction spatiale et discursive associe la désintégration physique du Chaâba à la désintégration morale dont on soupçonne toujours la communauté immigrée. La découverte de la boucherie clandestine de Saïd n'aide pas à redresser l'image des Maghrébins, toujours soupçonnés d'être hors-la-loi. La police ne se prive pas de sévir à la suite de cette activité qui fait la une de la presse locale. On le voit, avec ces manchettes dans les journaux, tout comme lorsqu'il s'agit de l'école où se rendent Omar et les autres, l'écriture, le «papier» est, pour l'immigrant, le symbole de l'aliénation et de la répression.

Dans un tel contexte, on en arrive très facilement aux syntagmes figés, expressions par excellence du stéréotype, sorte d'étiquettes qui enferment les immigrants dans une définition dépréciative: sale bicot, fainéant, bourricot sont leurs attributs les plus fréquents. Même la mauvaise connaissance ou l'ignorance du français est considérée comme une feinte par un agent des forces

20. Michel Foucault, Surveiller et punir, Paris, Gallimard, 1975, p. 229. 
de l'ordre ${ }^{21}$. C'est ainsi que le policier interprète l'inaptitude de madame Bouzid à s'exprimer dans cette langue comme un choix tactique délibéré, destiné à couvrir le crime d'un compatriote. Il dévoile, ce faisant, l'un des préjugés dont on affuble les Arabes et qui exprime l'une des plus grandes craintes des Occidentaux: ils sont d'une solidarité inébranlable, même dans le crime. Le réalisateur nous le fait voir par les échanges de repas, fréquents dans le film. Ainsi perçus, les immigrants d'Algérie apparaissent, pour les Français, comme une menace sérieuse, comparable à une épidémie.

Cette comparaison est en effet induite par certaines des techniques cinématographiques déployées par le réalisateur, mais aussi et surtout par des images, absentes du roman, qu'il incorpore au film. Sur le chemin de l'école, sur le tas d'ordures ou simplement dans la cour, les gones sont généralement une bande en mouvement, filmés en plongée. Le film, plus que le roman, donne ainsi l'impression d'un raz-de-marée impétueux ou d'un troupeau solidaire en transhumance. En tout cas, pour la société française, la nécessité d'une digue s'impose d'autant plus que le désir d'aseptiser son espace est nourri par les images que l'on associe aux Arabes et qui sont celles de rats et de chiens. Les rats qui sortent de partout au Chaâba, servant parfois de compagnons de jeu aux gones, et dont Nasser Bouafia et Sélim Bouchaoui introduisent quelques échantillons dans la classe de M. Grand, ne sont même pas évoqués dans le roman. Bêtes indésirables parce que prolifiques et dévastatrices, les rats symbolisent avant tout la clandestinité. Azouz Begag remarque d'ailleurs que cette image est associée aux Arabes "pour souligner consciemment ou inconsciemment leur présence "rampante" et "envahissante" dans la société ${ }^{22} »$. Les rats évoquent, en outre, les maladies infectieuses telles que la peste, comme chez Albert Camus ${ }^{23}$ par exemple, et les chiens, la rage. Ceux de Ruggia sont errants, laissés sans soins et donc plus dangereux. Ils apparaissent vers la fin du film comme

21. Ce n'est pas entièrement faux. Aux Antilles, par exemple, on sait que les esclaves utilisaient la langue comme stratégie de défense, ce qui déroutait complètement les esclavagistes.

22. Azouz Begag et Christian Delorme, Quartiers sensibles, Paris, Seuil, 1994, p. 8.

23. Dans La peste (Paris, Gallimard, 1947), roman d'Albert Camus, les rats sont les vecteurs de l'épidémie de la peste qui frappe la population d'Oran, en Algérie. Cette épidémie elle-même est la métaphore de la pourriture morale. Le médecin qui la combat se rend à l'évidence que «la meilleure façon de lutter contre la peste, c'est l'honnêteté» (p. 180). 
s'ils prenaient au Chaâba la place laissée par les gones. Eux aussi sont une figure de l'Arabe, si l'on s'en tient à cet autre roman de Begag ${ }^{24}$. Bref, selon la perspective de Ruggia, les Français perçoivent les Arabes du Maghreb comme un fléau grave dont il faut se protéger - et la meilleure prévention contre les épidémies infectieuses est, précisément, la mise en quarantaine ${ }^{25}$. Dans un tel contexte, on comprend que, dans le roman comme dans le film, être immigré désigne une expérience du malheur.

\section{Du malheur d'être immigré}

Kristeva posait déjà avec insistance cette question, dont la portée est immense: «Pourrons-nous intimement, subjectivement, vivre avec les autres, vivre autres, sans ostracisme, mais aussi sans nivellement ${ }^{26}$ ? " C'est connu, l'ostracisme est l'un des motifs récurrents des «littératures de l'immigration» et Le gone $d u$ Chaâba, roman et film, n'échappe pas à cette rhétorique. De nombreux mécanismes sont, en effet, mis en branle pour entraver les mouvements des Maghrébins. La barrière qui encercle le Chaâba, sa situation au seuil de la forêt, nature sauvage, sont destinées à les maintenir à distance selon un principe bien simple, celui de «la localisation élémentaire et du quadrillage. À chaque individu, sa place; et en chaque emplacement, un individu ${ }^{27} »$. Il faut remarquer que le réalisateur ne montre pas du tout le mur d'enceinte de l'école, mais insiste sur celui qui entoure le Chaâba. Ne peut-on pas y lire le fait que les Français, en situation de domination, ne veulent admettre aucun mélange, aucune concession? N'est-ce pas une manière de protéger la "pureté » de la culture française contre un environnement qui est une réserve, un bantoustan et un dépotoir? Fanon nous avertissait déjà : «L'indigène est un être parqué,

24. Azouz Begag, Les chiens aussi, Paris, Plon, 1995. C'est à partir de l'affiche haineuse «INTERDIT AUX ARBRES ET AUX CHIENS» interdisant l'accès des cafés et restaurants français aux Algériens pendant la guerre d'Algérie que Begag a conçu le personnage du chien pour représenter l'Arabe.

25. Dans son roman, L'opium et le bâton (Paris, Plon, 1965), l'Algérien Mouloud Mammeri compare la marginalisation des Arabes par les Français à «la fuite loin du virus et de la contagion»(p. 34). De même, dans La Québécoite (Montréal, XYZ éditeur, 1993, p. 54), la romancière Régine Robin considère l'établissement des immigrants dans les quartiers périphériques de Montréal comme une mise en quarantaine, justement.

26. Julia Kristeva, Étrangers à nous-mêmes, ouv. cité, p. 10.

27. Michel Foucault, Surveiller et punir, ouv. cité, p. 168. 
l'apartheid n'est qu'une modalité de la compartimentation du monde colonial. La première chose que l'indigène apprend, c'est de rester à sa place ${ }^{28} »$. Cela ne semble que trop vrai pour cette France des immigrés où les barrières ne sont pas seulement physiques.

Ces barrières géographiques sont également renforcées par d'autres, plus subtiles: la barrière linguistique que représente la langue française et la barrière psychologique, avec tous les stéréotypes qui enferment les Arabes dans des attitudes sclérosées, des professions abhorrées des Français et le statut d'élèves de seconde zone. Dans le film, la belle Jasmine, "prisonnière du Chaâba», et les deux petits poissons de Hacène et Omar enfermés dans une vieille télévision sont la métaphore de cette claustration subie par les immigrants algériens en France. Les Arabes sont confinés aux marges de la société parce que leur étrangeté est perçue défavorablement. Sous l'impulsion d'un réflexe de survie procédant d'une peur qui, selon Edward W. Said ${ }^{29}$, est cultivée en Europe depuis des lustres, les Français recourent à la distanciation et transforment en différence radicale des traits n'ayant aucune valeur intrinsèque. Le réalisateur semble ainsi pointer du doigt cette attitude qui consiste, pour les Français, à ne reconnaître de valeurs que les leurs. Le droit à la différence est dénié à l'immigrant. On pourrait dire que le film est un réquisitoire contre l'ethnocentrisme, cette tendance "à ériger, de manière indue, les valeurs propres de la société à laquelle j'appartiens en valeurs universelles ${ }^{30}$ ». C'est placé sous ce jour, en effet, qu'on pourrait lire le compte rendu de lecture que fait Omar à son cousin Hacène (14 minute).

Cette séquence, allégorie d'une vie d'immigrant, ne figure pas dans le roman et si Ruggia l'ajoute au film, c'est fort probablement pour orienter la démarche herméneutique. Assis seul dans un coin de la cour, un livre à la main, mais les yeux fermés, Omar explique à Hacène, pour justifier sa curieuse attitude, l'histoire du livre. Il y est question d'un homme enfermé dans une maison pour avoir écrit un livre. Ses gardiens menacent de lui tirer une balle dans la tête chaque jour, mais ne s'exécutent jamais. Ce qu'ils veulent, en

28. Frantz Fanon, Les damnés de la terre, ouv. cité, p. 18.

29. Edward W. Said, L'orientalisme. L'Orient créé par l'Occident, nouvelle édition augmentée, Paris, Seuil, 1997, p. 292.

30. Tzvetan Todorov, Nous et les autres, Paris, Seuil, 1989, p. 19. 
fait, c'est le rendre fou, non le tuer. L'homme en est conscient et ne se laisse pas déstabiliser; il ferme les yeux et pense à un autre monde. Cette histoire est une parabole de celle des immigrants harcelés et acculés à la marginalisation par la communauté d'accueil. L'étrangeté de l'immigrant, tout comme la folie de l'homme en question, sont l'œuvre de la société, une construction discursive, comme dirait Foucault ${ }^{31}$. Sous ce rapport, les modifications apportées au texte du roman par le réalisateur semblent plus motivées par une prise de position idéologique que par les exigences du média cinématographique. D'ailleurs, si l'on songe au maniement de la caméra qui produit de gros plans et des plans longs, on n'hésiterait pas à ranger Le gone du Chaâba parmi les films qui sont marqués par la personnalité du cinéaste, dont la visibilité est manifeste. Selon Manthia Diawara, ces distances focales, qui ne sont pas du tout gratuites, sont plutôt une marque de subjectivité: "L'utilisation de plans rapprochés de visages et d'objets dans des films où les longs plans dominent la narration révèle, de la part du réalisateur, une intervention didactique ${ }^{32}$.»

Mais si Ruggia construit un discours français univoque face à l'immigration, construction très partiale d'ailleurs, il procède différemment pour les Maghrébins. Bien que minoritaires et marginalisés par le fait même de leur extraterritorialité, ils n'en considèrent pas moins les Français comme autres. Mais ici, plusieurs tendances traversent le groupe car, ainsi que le dit Paterson: "Tributaire d'un processus de construction idéologique, toute altérité est variable, mouvante et susceptible de renversements. Elle n'est marquée d'aucune immanence et peut être dotée de traits positifs ou négatifs, euphoriques ou dysphoriques, dans un même espace social ou discursif ${ }^{33}$ ».

Ruggia prélève du groupe algérien du roman trois familles qu'il met en vedette, illustrant par ce discours dialogique différentes facettes de l'immigration. Les familles Bouchaoui, Saïd et Bouzid ne se font pas d'illusion. Elles se savent exclues ou rejetées

31. Michel Foucault, Folie et déraison. Histoire de la folie à l'âge classique, Paris, Plon, 1961 ; édition augmentée, Paris, Gallimard, 1972.

32. Manthia Diawara, "Popular Culture and Oral Traditions in African Films", dans Imruh Bakari et Mbye Cham (sous la dir. de), African Experiences of Cinema, London, British Film Institute, 1996, p. 215: "The use of close-ups, of human faces and of objects, in [films] where long shots dominate the narration, reveals a didactic intervention on the part of the director"

33. Janet M. Paterson, "Pour une poétique du personnage de l'autre», art. cité, p. 106. 
par la société française et, en même temps, coupées de leur terroir algérien. Elles développent pourtant des stratégies de survie différentes. Les Saïd et les Bouchaoui ont apparemment abandonné tout espoir d'intégration à la société d'accueil. M. Bouchaoui, nostalgique et en butte aux échecs de l'heure, se tourne vers le passé, car il est incapable d'assumer sa présence en France. Il regrette amèrement d'avoir quitté El-Ouricia. Mais comme le relève Kristeva, «le paradis perdu est un mirage du passé qu'il [l'étranger] ne saura jamais retrouver ${ }^{34} »$. Par conséquent, il rumine son «mal du pays», quand il le peut, ou l'exprime, comme tous ses congénères d'ailleurs, par la violence exercée contre les enfants.

Saï, comme son nom l'indique, se contente de la place que lui fait la France. Il assume, sans ambitions visibles, les stéréotypes qu'on lui colle. Toujours rivé à son émission en langue arabe, il ne fait aucun effort pour soutenir la réussite scolaire de ses enfants. Il les encourage plutôt à mener des activités "dégradantes», telles que le petit commerce, auxquelles on identifie les immigrants pour mieux les dévaloriser. Il n'est d'ailleurs pas prêt à mettre fin à la boucherie clandestine. Rabah, son fils aîné, à qui il donne une mobylette et un poste de manœuvre, perpétuant ainsi le préjugé socioprofessionnel, est un bon chef de gang, image type de l'enfant de banlieue. Hacène, son autre fils, est un souffre-douleur contre qui il retourne toute sa rage d'immigrant désabusé, comme si les échecs scolaires de l'enfant étaient le symptôme principal de son exil manqué. En fait, il contribue lourdement à cet échec et on a l'impression que les sévices qu'il inflige à son fils relèvent d'une autoflagellation car, comme le souligne si bien Kristeva, l'immigrant mélancolique s'en prend à tout le monde, y compris à luimême.

Hacène, Sélim Bouchaoui et Nasser éprouvent le même désarroi que leurs parents. En butte à une société qu'ils se représentent fermée, agressive, hypocrite et hostile, ils se sentent méprisés et ignorés. En classe, ils occupent le fond, appelé "coin des bourricots». Le maître, qui ne tolère aucun écart de leur part, n'a pas d'égard pour la prononciation de leur nom; ils sont régulièrement rappelés à l'ordre, insultés ou tournés en dérision. Ce manque de reconnaissance suscite chez eux une haine violente des Français et de tous ceux qui s'en rapprochent. Bouchaoui manque de respect à $M$. Grand et le traite de raciste. Nasser et lui

34. Julia Kristeva, Étrangers à nous-mêmes, ouv. cité, p. 20. 
introduisent des rats en classe et commettent des voies de fait sur Omar. Ils se donnent par là les moyens d'une auto-affirmation car, comme le rappelle Kristeva,

[d] ans l'univers d'esquives et des faux-semblants qui constituent ses pseudo-rapports aux pseudo-autres, la haine procure à l'étranger une consistance. C'est à cette paroi douloureuse mais sûre et, en un sens, familière, qu'il se heurte pour s'affirmer présent aux autres et à lui-même. La haine le rend réel, authentique en quelque sorte, solide ou, simplement, existant ${ }^{35}$.

Hacène est habité par la même haine, mais ne parvient pas à l'exprimer. Totalement déboussolé, il ne peut trouver de réconfort nulle part, ni à l'école, ni en famille. L'angoisse et la haine ruminées se muent en névrose. L'air absent, comme pour nier la réalité, il détruit, avec des gestes d'automate, ses fournitures scolaires, plante violemment sa plume dans la main du maitre et essaye de se pendre. Par la suite, à la vue des policiers venus quérir le boucher clandestin, son père, il entonne son incompréhensible refrain: «Je ne veux pas être guillotiné », et Omar le dira "un peu fou». Cette scène, qui ne figure pas dans le roman de Begag, est introduite ici pour faire pendant au compte rendu de lecture d'Omar que l'on a évoqué plus haut. La folie de Hacène est le fait de la société française, dont la guillotine symbolise l'intransigeance, mieux, l'intolérance. Ruggia parvient ainsi à déconstruire un préjugé tenace qu'on associe aux immigrants. La violence, la dépression et la folie dont on les accuse souvent ne sont pas du tout consubstantielles à leur nature ou à leur nationalité. Ce sont, au contraire, des attitudes conjoncturelles suscitées par l'action et le discours du groupe d'accueil. Ce film reprend dès lors la mise en garde de Pierre Bourdieu, pour qui «il faut se garder de transformer en propriétés nécessaires et intrinsèques d'un groupe quelconque [...] les propriétés qui leur incombent à un moment donné du temps du fait de leur position dans un espace social déterminé, et dans un état déterminé de l'offre des biens et des pratiques possibles ${ }^{36} »$.

Mieux que Begag, Ruggia montre comment le contexte socioéconomique détermine les réactions psychologiques des individus et des groupes, ce qui n'est pas sans rappeler le programme du naturalisme. Une première catégorie d'Algériens se laisse prendre

35. Julia Kristeva, Étrangers à nous-mêmes, ouv. cité, p. 24.

36. Pierre Bourdieu, Raisons pratiques. Sur la théorie de l'action, Paris, Seuil, 1994, p. 19. 
au piège de la marginalisation en l'assumant sans recul et dans la souffrance. Bouzid et son fils donnent corps à la deuxième tendance. La famille Bouzid, en effet, est déterminée à rompre la chaîne des stéréotypes avec lesquels on cherche à la lier. La France, le luxe qu'elle offre, les professions autres que manuelles représentent le modèle, l'idéal à atteindre. Dans le schéma des types d'altérité de Landowski ${ }^{37}$, les Bouzid correspondent, par leur trajectoire ascensionnelle, au snob ou, dans une moindre mesure, au dandy dont les efforts pour se rapprocher du groupe dominant relèvent d'un savant calcul: il faut masquer tout ce qui distingue et adopter ce qui fait corps avec la culture d'accueil. Il s'agit, avant tout, de détruire les stéréotypes. Le réalisateur leur donne un coup de main en refaisant le portrait de Bouzid qui, du coup, prend plus d'épaisseur psychologique. Il le montre soucieux de paraître conforme à la norme française, même si cela ressemble fort à de l'assimilation. En dépit du fait qu'il ne comprend pas la langue française, il refuse obstinément de se faire traduire le journal en arabe, proclamant avec force sa bonne maîtrise de cette langue de l'élite. C'est, en fait, le processus de l'aliénation/assimilation décrit par Fanon ${ }^{38}$ qui est en cause, en tant qu'adhésion totale et inconditionnelle du colonisé à de nouveaux modèles culturels, au détriment de son propre univers de référence.

Par ailleurs, Ruggia hypertrophie la volonté de succès des Bouzid, succès qui passe par l'école, puisque ici on mise sur le capital culturel. Le livre devient, dans son film, un motif primordial. À l'occasion de la circoncision, Omar reçoit un livre et, pendant que ses camarades se disputent de vieilles chaussures et d'autres bibelots prodigués par la poubelle, il se délecte des livres qu'ils ont foulés au pied. Le livre étant le symbole culturel par excellence, l'attachement qu'il suscite signifie aussi l'acceptation de l'assimilation qui est, paradoxalement, une forme d'intégration entraînant, en même temps, l'abandon de certaines pratiques et valeurs arabes ${ }^{39}$. Voilà pourquoi, par exemple, Omar refuse d'aller à l'école en gandoura. $\mathrm{Au}$ risque de passer pour des renégats, les Bouzid se dissocient de toutes les activités pouvant entraver leur entrée dans la société française: Omar refuse d'aider frauduleusement Nasser au nom de la

37. Éric Landowski, Présences de l’autre, ouv. cité, p. 52-55.

38. Frantz Fanon, Pour la révolution africaine, Paris, Maspéro, 1969, p. 39.

39. Cette forte valeur symbolique du livre et de l'écrit rappelle une scène du film Afrique je te plumerai (Jean-Marie Teno, 1991) où un jeune enfant va voler un livre dans une librairie. 
solidarité arabe; son père détruit la boucherie de Saïd, non sans l'avoir maudit. La destruction des pesanteurs et des symboles de la fixité est traduite au cinéma par les jeux de scène. Les courses folles d'Omar à travers le Chaâba, ses escapades à vélo et son désir insistant de déménager représentent métaphoriquement le rejet des stéréotypes, de l'immobilisme et, forcément, de toute une dimension de son arabité, ce qui est malheureux. Le réalisateur attribue à son protagoniste un rêve qui montre le Chaâba en feu, illustrant ainsi non seulement l'obsession du jeune Omar, mais aussi et surtout le dilemme profond des immigrants: peut-on s'intégrer aux autres sans se renier, ne serait-ce qu'en partie, et sans trahir les siens? On le voit, l'immigration est indissociable de la question de l'acculturation et de l'aliénation.

Multipliant les efforts, Omar se hisse au deuxième rang de sa classe, est cité en exemple par M. Grand et trouve place aux côtés de Jean-Marc Laville, rebaptisé Francis (comme la France?) par le réalisateur. Il reçoit en guise de récompense une image de Charlemagne, empereur des Francs et inventeur de l'école. Il s'agit en fait d'un certificat d'admission à la culture française, culture élitiste qui n'admet que les postulants ayant fait leurs preuves. La seule voie d'intégration ouverte à l'immigrant reste l'école et, semble dire Ruggia, il ne lui suffit pas de se fondre dans la majorité, il doit se distinguer, se hisser au sommet. Dans un tel contexte, seules ses capacités intellectuelles peuvent le sauver; Kristeva ne dit pas autre chose:

Enfin, lorsque votre étrangeté devient une exception culturelle - si, par exemple, vous êtes reconnu comme un grand savant ou un grand artiste - la nation toute entière annexera votre performance, l'assimilera à ses meilleures réalisations et vous reconnaitra mieux qu'ailleurs, non sans un certain clin d'œil concernant votre bizarrerie si peu française ${ }^{40}$.

Mais un immigrant qui change de statut est confronté à un nouveau type de relation avec ceux d'entre ses compatriotes qui n'ont pas su ou pas pu franchir le pas. La distance se crée entre eux et fait naître la suspicion. Voilà pourquoi Omar est accusé de trahison et mis au ban du groupe. Il connaît la solitude et la mélancolie lorsqu'on l'accuse d'être français à cause de son intelligence et des traitements de faveur dont il bénéficie. Cependant, là où le roman se contente de poser un problème, le film suggère une

40. Julia Kristeva, Étrangers à nous-mêmes, ouv. cité, p. 60. 
solution. Chez Ruggia, intégration ne rime pas du tout avec reniement. En plus du remords qui habite Omar lorsque son arabité est mise en cause, il semble investi d'une mission de leader. Attaché à ses racines arabes, il s'écarte remarquablement du personnage individualiste du roman. Là, il refuse son aide à Nasser parce que, pense-t-il, tout le monde ne peut pas être premier; ici, il ne rejette que la forme que cette aide pourrait prendre. On le voit animé du désir de tirer les autres par le haut, bien sûr, sans se compromettre. C'est ainsi qu'il prend la défense de ses concitoyens pendant le cours d'hygiène, qui tourne mal. Il sauve Hacène du suicide et, lorsqu'il conduit les policiers à la boucherie de Saïd, il ne s'agit pas d'un acte de bravoure et de vanité comme dans le roman: il le fait plutôt pour sauver son cousin de la guillotine. Au moment de leur départ du Chaâba, il lui offre un viatique pour la civilisation française: le dictionnaire découvert dans la poubelle. Pour Ruggia, en somme, l'immigrant doit rechercher un certain équilibre entre deux communautés. S'il s'écarte de son peuple, c'est pour lui revenir, tel Prométhée, enrichi et plus utile. Autant M. Grand représente la France, autant Omar représente l'idéal de l'immigrant. Il doit être ambitieux et s'élever au-dessus des contraintes sociales et vers de nouveaux horizons, comme le rêveur de "Liberté», poème de Maurice Carême déclamé par le jeune garçon dans le film. Seulement, il devra prendre conscience de son rôle de porte-fanion de sa communauté d'origine, devenu garant de leur destin collectif. Cela lui permet, et on le voit avec Omar, de négocier ce que Homi Bhabha appelle un "tiers-espace» (third space), lequel l'aide à éluder les bipolarités traditionnelles marquant les rapports entre centre et périphérie, colonisateur et colonisé.

Du livre à l'écran, Le gone du Chaâba a subi de nombreuses transformations. Les suppressions et compressions des personnages et des séquences, de toute évidence, ne sont pas uniquement destinées à assurer la translation du texte d'un média à l'autre. Ruggia s'est servi du roman de Begag comme d'une amorce pour formuler sa propre vision de l'immigration. Sans contredire le roman, le film procède par grossissements, mettant l'accent sur des aspects simplement évoqués dans le livre. Ruggia parvient ainsi à peindre, avec une précision digne des naturalistes, la situation précaire de l'immigrant. Marginalisé par une culture occidentale ethnocentrique, il peut, s'il n'y prend garde, sombrer dans la violence et la dépression.

L'école, enfin, est présentée comme l'unique voie de salut, mais le réalisateur recommande persévérance et clairvoyance aux 
immigrants. Ils doivent négocier leur insertion dans leur communauté d'accueil et éviter les pièges de l'aliénation en gardant de solides attaches avec leur groupe d'origine.

Au-delà de la fidélité à l'auteur, au texte ou au média, la réécriture filmique de Ruggia est davantage de l'ordre d'une réinvention permanente de sens. Il emprunte, on l'a vu, non seulement au Gone du Chaâba, mais aussi à d'autres textes du même auteur, voire à ceux d'autres romanciers et à la poésie une matière à laquelle il insuffle son génie propre, conférant ainsi une nouvelle vie à chacune de ses sources. 